\title{
Multisystem inflammatory syndrome with refractory cardiogenic shock due to acute myocarditis and mononeuritis multiplex after SARS-CoV-2 infection in an adult
}

\author{
Othenin-Girard Alexandra ${ }^{a b^{*}}$, Regamey Julien ${ }^{c^{*}}$, Lamoth Frédéric ${ }^{\text {def }}$, Horisberger Alice ${ }^{\mathrm{g}}$, Glampedakis Emmanouil ${ }^{\mathrm{d}}$, Epiney \\ Jean-Benoit ${ }^{\mathrm{h}}$, Kuntzer Thierry ${ }^{\mathrm{fh}}$, de Leval Laurence ${ }^{\mathrm{fi}}$, Carballares Maudej, Hurni Claire-Anne ${ }^{\mathrm{a}}$, Rusca Marco ${ }^{\mathrm{a}}$, Pantet Olivier $^{\text {af }}$, \\ Di Bernardo Stefano ${ }^{\mathrm{fk}}$, Oddo Mauro ${ }^{\text {af }}$, Comte Denis ${ }^{\mathrm{fg} \dagger}$, Piquilloud Lise $^{\text {aft }}$
}

a Adult Intensive Care Unit, Lausanne University Hospital, Lausanne, Switzerland

b Anaesthesiology Service, Lausanne University Hospital, Lausanne, Switzerland

c Cardiology Service, Lausanne University Hospital, Lausanne, Switzerland

d Infectious Diseases Service, Lausanne University Hospital, Lausanne, Switzerland

e Institute of Microbiology, Lausanne University Hospital, Lausanne, Switzerland

f University of Lausanne, Faculty of Biology and Medicine, Lausanne, Switzerland

g Immunology and Allergy Service, Department of Medicine, Lausanne University Hospital, Lausanne, Switzerland

h Neurology Service, Department of Clinical Neurosciences, Lausanne University Hospital, Lausanne, Switzerland

Institute of Pathology, Department of Laboratory Medicine and Pathology, Lausanne University Hospital, Lausanne, Switzerland

Emergency Medicine Service, Lausanne University Hospital, Lausanne, Switzerland

k Paediatric Cardiology Service, Department of Woman-Mother-Child, Lausanne University Hospital, Lausanne, Switzerland

Equal first authors

Equal last authors

Correspondence:

Dr Lise Piquilloud, MD,

PhD, Adult Intensive Care

Unit, Lausanne University

Hospital (CHUV), Rue du

Bugnon 46, CH-1011 Lau-

sanne, lise.piquil-

loud[at]chuv.ch

\section{Summary}

A 22-year-old male with a typical history of pauci-symptomatic COVID-19 3 weeks earlier, confirmed by positive serology for SARS-CoV-2 $(\lg G)$, was admitted to the intensive care unit because of severe myocarditis with refractory cardiogenic shock that required extracorporeal life support. Due to a clinical presentation suggestive of Kawasaki-like disease with coronary aneurysm and severe systemic inflammation, intravenous immunoglobulins were administered in combination with tocilizumab. The initial clinical course was favourable with these treatments. However, the patient subsequently developed a severe mononeuritis multiplex leading to bilateral foot drop, which required intensive immunosuppressive therapy (corticosteroids, cyclophosphamide and rituximab). The clinical presentation meets the criteria for multisystem inflammatory syndrome associated with SARS-CoV-2, but includes very severe organ damages. Early recognition, a multidisciplinary approach and aggressive therapeutic intervention can lead to a favourable outcome.

Keywords: cardiogenic shock, COVID-19, Kawasaki disease, mononeuritis multiplex, multisystem inflammatory syndrome, myocarditis, SARS-CoV-2

\section{Introduction}

Coronavirus disease 2019 (COVID-19), due to the emerging SARS-CoV-2 virus, was first described in December 2019 as clusters of severe pneumonia [1]. Recently, a mul- tisystem inflammatory syndrome likely linked to SARSCoV-2 and which shows similarities with Kawasaki disease [2] has been described in children (MIS-C) [3], and, rarely, in adults [4]. Most affected patients had negative SARS-CoV-2 polymerase chain reaction (PCR) on nasopharyngeal swab, but the presence of specific IgG antibodies in blood suggests an auto-immune response following pauci-symptomatic COVID-19 [2]. We describe a case not in a child or adolescent, but in an adult, of severe multisystem inflammatory syndrome (MIS) associated with SARS-CoV-2 featuring Kawasaki-like disease and severe myocarditis with refractory cardiogenic shock that required extracorporeal life support. Our patient also developed severe mononeuritis multiplex, which has not been previously described as related to SARS-CoV-2 infection.

\section{Case description}

A healthy 22-year-old male patient of East African descent was admitted because of a five-day history of asthenia, chills, diffuse myalgia, abdominal pain and diarrhoea. The day before admission, he developed a non-pruriginous rash, a non-productive cough and odynophagia. He described transient anosmia and dysgeusia three weeks before admission. Maculopapular rash, targetoid skin lesions of the trunk and extremities, bilateral palmar redness and pharyngeal erythema were observed at clinical examination. A signs and symptoms timeline is displayed in figure 1. The chest and abdominal computed tomography scan 
showed segmental pulmonary embolism with normal lung parenchyma, and mesenteric lymphadenitis. The nasopharyngeal PCR was slightly positive for SARS-CoV-2. SARS-CoV-2 serology was positive (IgG enzyme-linked immunosorbent assay (ELISA)). Blood biomarkers revealed severe inflammation (C-reactive protein at $275 \mathrm{mg}$ / 1, white blood cell count $18.2 \mathrm{G} / \mathrm{l}$, fibrinogen $8.5 \mathrm{~g} / \mathrm{l}$ ), elevated D-dimers $(3322 \mathrm{ng} / \mathrm{ml})$ and increased creatinine $(150 \mu \mathrm{mol} / \mathrm{l})$. Screening for autoimmune diseases (antineutrophil cytoplasmic antibodies, rheumatoid factor, antinuclear antibodies) was negative.

Within 12 hours, the patient developed fever (maximum $40.2^{\circ} \mathrm{C}$ ) and refractory cardiogenic shock due to severe biventricular dysfunction without pericardial effusion that promptly required intubation and veno-arterial extracorporeal membrane oxygenation (VA-ECMO). High-sensitive troponin $\mathrm{T}(2718 \mathrm{ng} / \mathrm{l})$ and creatinine kinase (768 U/ 1 , MB fraction 16\%) were increased. An electrocardiogram showed no signs of acute myocardial ischemia and a diagnosis of acute myocarditis was retained. In accordance with the local standard of care for severe COVID-19, an initial dose of tocilizumab $(8 \mathrm{mg} / \mathrm{kg})$ was administered. Standard microbiological and immune panels for common causes of myocarditis were negative. An endomyocardial biopsy revealed a severe myocardial inflammation with several foci of myocyte necrosis (fig. 2). Biopsies of two inguinal lymph nodes showed non-specific signs of inflammation. The biopsy of an adjacent arteriole was normal. As an early complication, the patient developed a third-degree atrioventricular block that required temporary pacing. A transient ST segment elevation in the anterolateral leads justified a coronary angiogram that identified an aneurysm of the proximal left anterior descending coronary artery.

The diagnosis of MIS [3] with features of Kawasaki disease [5] related to SARS-CoV-2 infection was made and intravenous immunoglobulin therapy (IVIG, $2 \mathrm{~g} / \mathrm{kg} / 24$ hours) was started in accordance with guidelines for the management of Kawasaki disease [5]. A second dose of tocilizumab $(8 \mathrm{mg} / \mathrm{kg})$ was also administered. The rash resolved within 24 hours after IVIG treatment. The cardiac dysfunction rapidly recovered, allowing VA-ECMO removal on day six since admission, and the patient was extubated on day nine. The evolution over time of the biological markers is summarised in figure 3.

Five days after extubation, we noticed a severe right facial nerve palsy, bilateral intrinsic hand muscle weakness, bilateral foot drop and dysaesthesia of the four extremities. Nerve conduction studies performed on day 17 revealed normal motor responses after stimulation of the right median and both ulnar and tibial nerves, reduced amplitude of the motor response following right facial nerve stimulation and no response after bilateral stimulation of the peroneal nerve. There was a low amplitude sural sensory response. The cerebrospinal fluid showed an absence of white cells and slightly elevated proteins $(637 \mathrm{mg} / \mathrm{l}$, normal $<450$ ), without oligoclonal bands. The complete PCR panel for infectious causes of meningoencephalitis, including viral diseases and tuberculosis, was negative, as was SARS-CoV-2 PCR. Antineutrophil cytoplasmic, antineuronal and paraneoplastic antibodies were negative in serum and/or cerebrospinal fluid. Magnetic resonance imaging of the brain and spine did not show any ischaemic, haemorrhagic or inflammatory lesions. The overall clinical picture was consistent with a severe mononeuritis multiplex, and the patient received high-dose intravenous methylprednisolone ( $1 \mathrm{~g}$ /day for 5 days), followed by oral prednisone $(1 \mathrm{mg} / \mathrm{kg} / \mathrm{day})$ in combination with cyclophosphamide $(15 \mathrm{mg} / \mathrm{kg}) \quad[6,7]$. A second dose of cyclophosphamide $(15 \mathrm{mg} / \mathrm{kg})$ was administered on day 34. To avoid long-term complications of cyclophosphamide, rituximab $(1 \mathrm{~g})$ was then administered (days 37 and 55) $[6,7]$. Muscle and nerve (right sural nerve, gastrocnemius and deltoid muscle) biopsies did not show vasculitis, but neurogenic changes were found in the gastrocnemius muscle; the deltoid muscle biopsy was normal.

Figure 1: Signs and symptoms timeline.

Typical COVID-19 signs and symptoms

Evolution

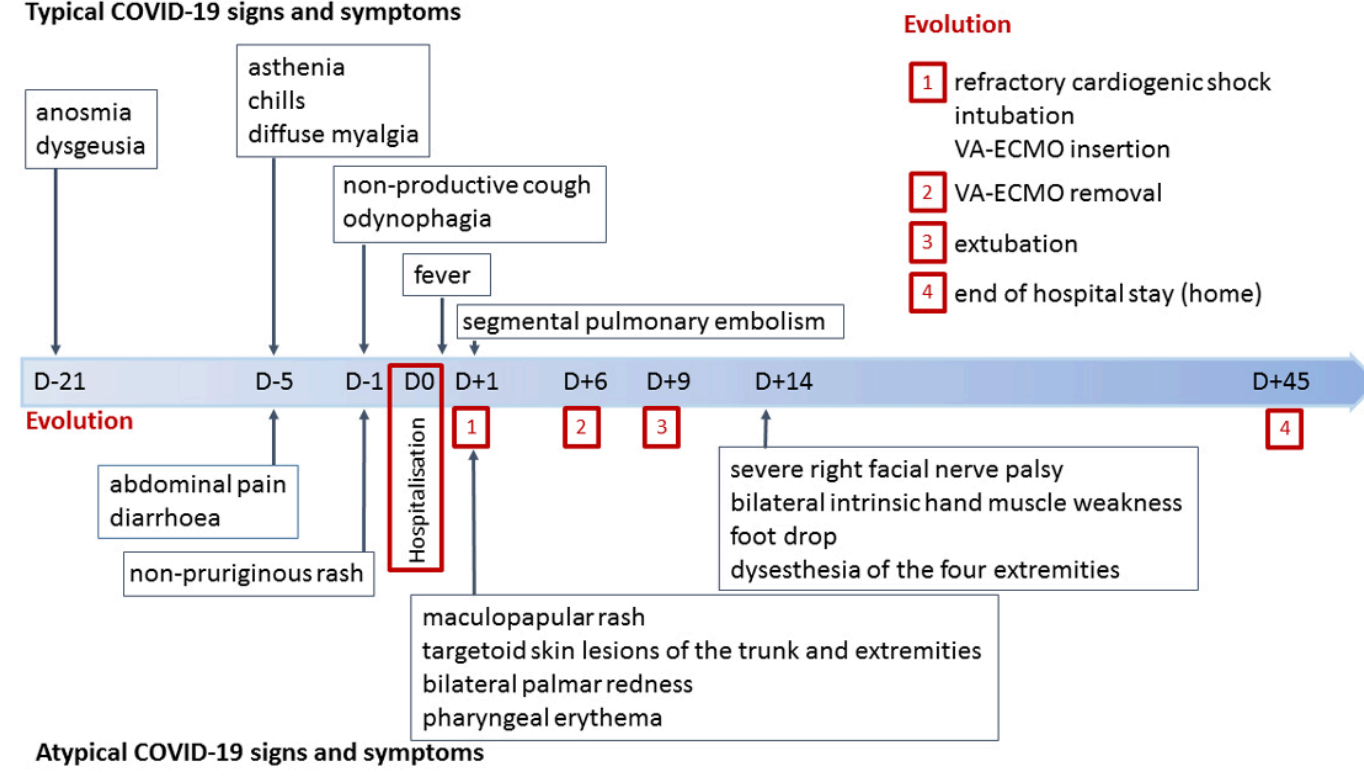

\section{asthenia}

chills

diffuse myalgia

odynophagia

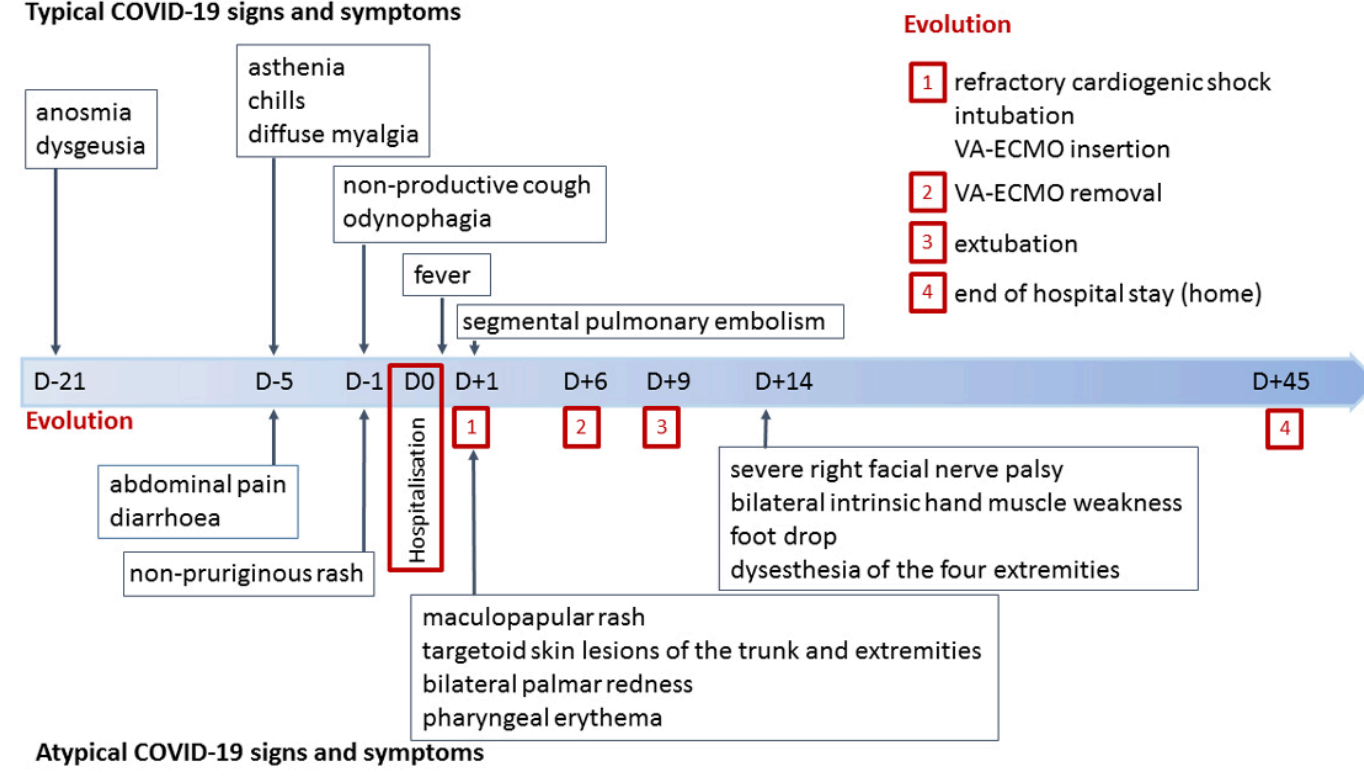


The patient returned home on day 45 with complete recovery of facial nerve palsy and slight improvement of peroneal paralysis. Four months later, the patient could walk without orthosis, but had persistent bilateral foot drop.

\section{Discussion}

We report a case of severe MIS in an adult patient following SARS-CoV-2 infection. The case was characterised by very severe cardiac and neurological damage that evolved favourably under treatment. The initial clinical presentation showed associated features of Kawasaki disease (fever, pharyngeal erythema, maculo-papular rash, palmar erythema and coronary aneurysm) [5] with cardiogenic shock due to severe myocarditis. Later, the patient was diagnosed with severe mononeuritis multiplex.

Kawasaki-like disease has been recently described in cases of MIS associated with SARS-CoV-2 infection in children and, rarely, in adults $[2,4]$. Consistent with the cardiovascular collapse described in Kawasaki disease shock syndrome (KDSS) [8], the aetiology of heart failure in our patient was the result of severe myocarditis. Myocarditis was probably the consequence of an immuno-inflammatory process, as suggested by the 3-week delay between first symptoms and the development of heart failure, the positive SARS-CoV-2 IgG serological test at the time of admission and the negative SARS-CoV-2 PCR in the endomyocardial biopsy. The subsequent neurological presentation of mononeuritis multiplex also suggests an immunemediated mechanism, even though the muscle and nerve biopsies did not show vasculitis, as is the case in about $40 \%$ of cases of immune-mediated vasculitic neuropathy [9]. Mononeuritis multiplex is a classic presentation of primary and secondary vasculitic neuropathies. Such a condition has been previously associated with other viruses, such as hepatitis B and C viruses and human immunodeficiency virus [6]. However, this is the first report to describe the development of mononeuritis multiplex in the context of SARS-CoV-2 infection.

The role of SARS-CoV-2 in the pathogenesis of MIS has not been elucidated to date. Recent studies have shown that SARS-CoV-2 can directly infect endothelial cells in different organs, including the lung, heart, kidney, liver and small intestine [10]. However, in our patient, PCR for SARS-CoV-2 was negative in the myocardial, lymph node, nerve and skin biopsies. In severe cases, SARS-CoV-2 induces excessive immune activation and an aberrant inflammatory response that shares common features with macrophage activation syndrome. Preliminary studies have shown an important increase of interleukin-6 in COVID-19 patients. It has been speculated that this cytokine plays a role in the pathogenesis of certain vasculitides (including Kawasaki disease) due to its effect on endothelial cells: it increases the number of adhesion molecules on the cell surfaces, enhances lymphocytes' adherence to the cells and promotes endothelial damage [11]. Moreover, complement activation and immune complexes depositions have been suggested as being responsible for the capillary inflammation $[12,13]$. $\mathrm{C} 3$ and $\mathrm{C} 4$ consumption was described in children with SARS-CoV-2-associated MIS-C [14] but was not present in our patient. Immunofluorescence staining for $\mathrm{C} 4 \mathrm{~d}$ was also negative in the endomyocardial biopsy. Finally, the presence of eosinophils and neutrophils in the endomyocardial biopsy reflects the importance of the innate immune system in the pathogenesis of organ damage linked to SARS-CoV-2 [15]. Interestingly, lymph node biopsies showed non-specific signs of inflammation. This is consistent with what has been previously described in Kawasaki disease unrelated to SARS-CoV-2 infection [16].

In cases of MIS and Kawasaki-like disease [2, 4], IVIG treatment is administered to all patients. Some patients also receive methylprednisolone [2]. Accordingly, we followed the treatment guidelines for Kawasaki disease and

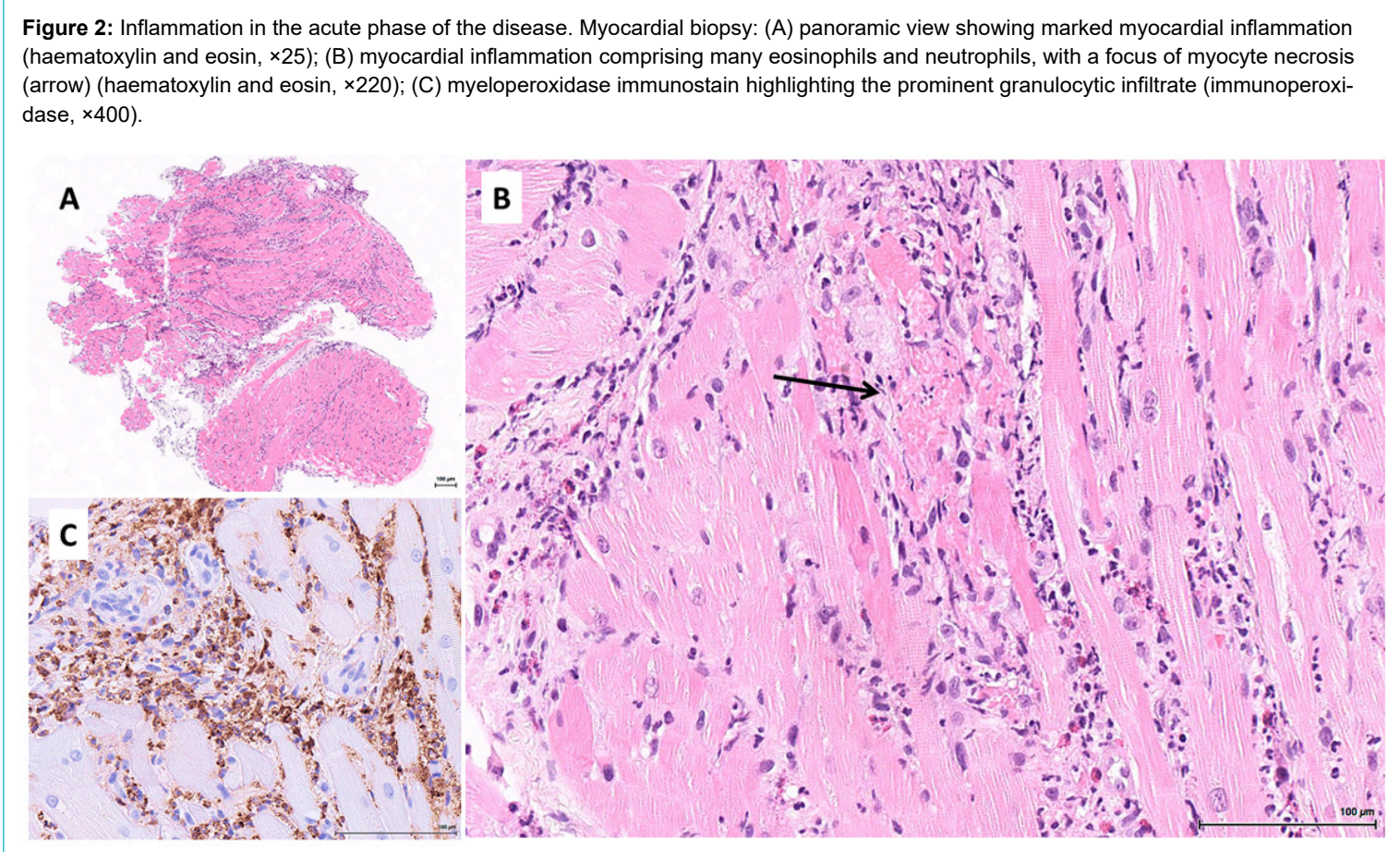


Figure 3: Evolution of the relevant biological markers over time: peripheral blood cell count (top panel), relative levels of the inflammation markers expressed as a ratio to the upper limit of the normal marker (middle panel), high-sensitive cardiac troponin T (bottom panel).TCZ = tocilizumab; IVIG = intravenous immunoglobulin; MPDN = methylprednisolone (arrow: first infusion of a five-day treatment); CYC = cyclophosphamide; $\mathrm{RTX}=$ rituximab

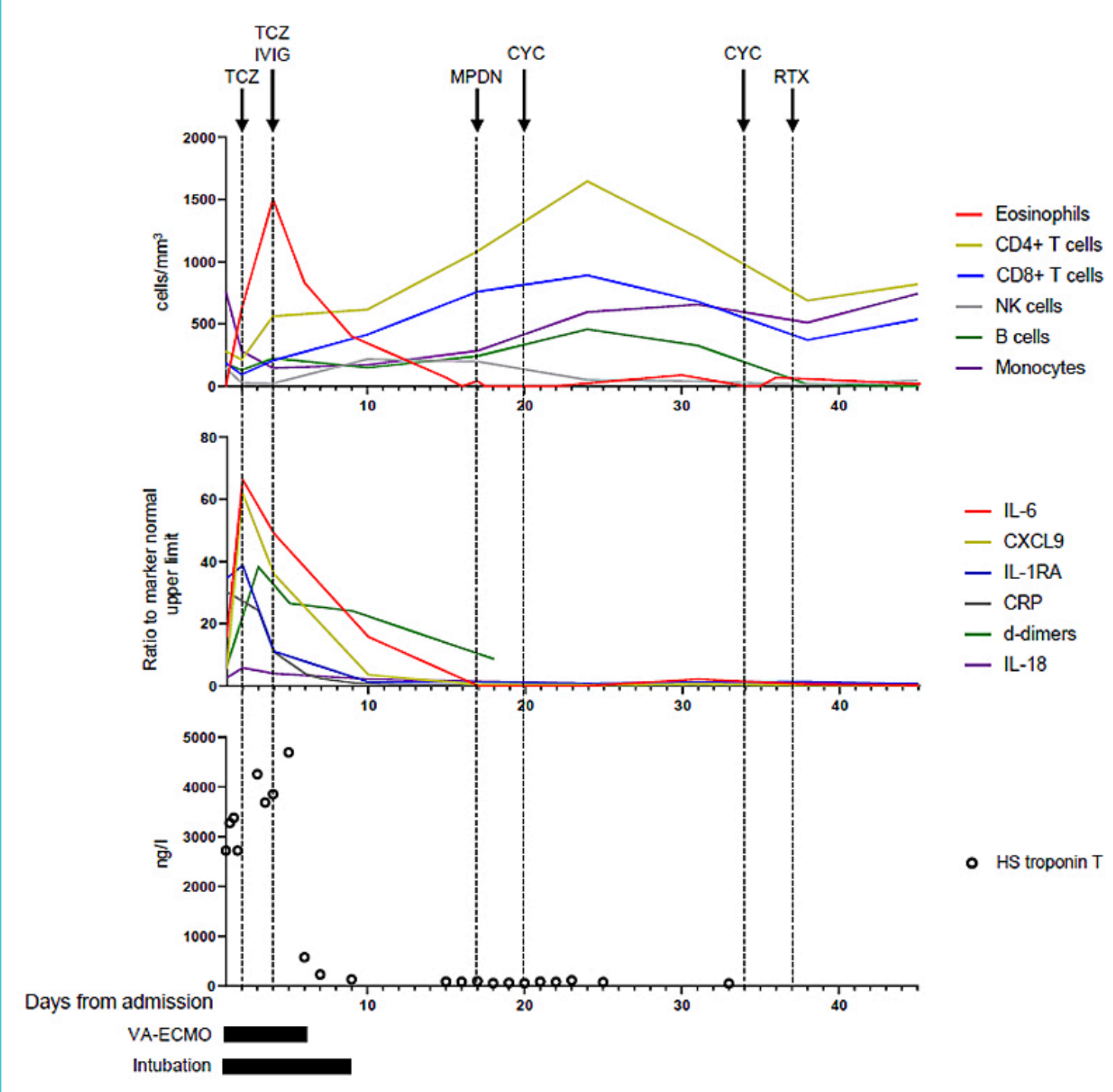

our patient received IVIG ( $2 \mathrm{~g} / \mathrm{kg}$, single dose) [5]. Preliminary data suggest that tocilizumab, an interleukin-6 receptor antagonist, may be useful to treat patients with severe COVID-19 [17], and therefore this treatment was also administered. The severe mononeuritis multiplex, demonstrated after extubation, justified additional treatment with high doses of glucocorticoids, cyclophosphamide, and then rituximab $[6,7]$. The clinical course was as follows: at hospital discharge, facial nerve palsy had fully recovered but bilateral peroneal paralysis had improved only slightly. Four months later, the patient could walk without orthosis, but had persistent bilateral foot drop.

In summary, MIS associated with SARS-CoV-2 can occur in adults. It can be referred to as MIS-A. The clinical presentation can include severe organ damage with features of incomplete or complete Kawasaki disease, including coronary aneurysm, as well as myocarditis-associated cardiogenic shock and also mononeuritis multiplex. This disease, which is probably immune-mediated, requires prompt diagnosis and treatment as it can be life threatening and may be associated with long-term cardiac and neurologic sequelae. In adult patients with MIS, peripheral neurological complications should be sought. In the absence of validated treatment recommendations, a multidisciplinary approach is recommended in order to assess the risk/benefit balance of adjunctive immune-modifying therapies.

\section{Disclosure statement}

No funding and no conflict of interest relevant to this article was reported.

\section{References}

1 Huang C, Wang Y, Li X, Ren L, Zhao J, Hu Y, et al. Clinical features of patients infected with 2019 novel coronavirus in Wuhan, China. Lancet. 2020;395(10223):497-506. doi: http://dx.doi.org/10.1016/ S0140-6736(20)30183-5. PubMed.

2 Verdoni L, Mazza A, Gervasoni A, Martelli L, Ruggeri M, Ciuffreda M, et al. An outbreak of severe Kawasaki-like disease at the Italian epicentre of the SARS-CoV-2 epidemic: an observational cohort study. Lancet. 2020;395(10239):1771-8. doi: http://dx.doi.org/10.1016/ S0140-6736(20)31103-X. PubMed.

3 Health Alert Network. Multisystem Inflammatory Syndrome in Children (MIS-C) Associated with Coronavirus Disease 2019 (COVID-19). 2020. 
Available from: https://emergency.cdc.gov/han/2020/han00432.asp [accessed 2020 June 9].

4 Shaigany S, Gnirke M, Guttmann A, Chong H, Meehan S, Raabe V, et al. An adult with Kawasaki-like multisystem inflammatory syndrome associated with COVID-19. Lancet. 2020;396(10246):e8-10. doi: http://dx.doi.org/10.1016/S0140-6736(20)31526-9. PubMed.

5 McCrindle BW, Rowley AH, Newburger JW, Burns JC, Bolger AF, Gewitz M, et al.; American Heart Association Rheumatic Fever, Endocarditis, and Kawasaki Disease Committee of the Council on Cardiovascular Disease in the Young; Council on Cardiovascular and Stroke Nursing; Council on Cardiovascular Surgery and Anesthesia; and Council on Epidemiology and Prevention. Diagnosis, Treatment, and LongTerm Management of Kawasaki Disease: A Scientific Statement for Health Professionals From the American Heart Association. Circulation. 2017;135(17):e927-99. doi: http://dx.doi.org/10.1161/

CIR.0000000000000484. PubMed.

6 Gwathmey KG, Burns TM, Collins MP, Dyck PJ. Vasculitic neuropathies. Lancet Neurol. 2014;13(1):67-82. doi: http://dx.doi.org/ 10.1016/S1474-4422(13)70236-9. PubMed.

7 Collins MP, Dyck PJ, Gronseth GS, Guillevin L, Hadden RD, Heuss D, et al.; Peripheral Nerve Society. Peripheral Nerve Society Guideline on the classification, diagnosis, investigation, and immunosuppressive therapy of non-systemic vasculitic neuropathy: executive summary. J Peripher Nerv Syst. 2010;15(3):176-84. doi: http://dx.doi.org/10.1111/ j.1529-8027.2010.00281.x. PubMed.

8 Gatterre $\mathrm{P}$, Oualha M, Dupic L, Iserin F, Bodemer C, Lesage F, et al. Kawasaki disease: an unexpected etiology of shock and multiple organ dysfunction syndrome. Intensive Care Med. 2012;38(5):872-8. doi: http://dx.doi.org/10.1007/s00134-012-2473-8. PubMed.

9 Collins MP, Mendell JR, Periquet MI, Sahenk Z, Amato AA, Gronseth GS, et al. Superficial peroneal nerve/peroneus brevis muscle biopsy in vasculitic neuropathy. Neurology. 2000;55(5):636-43. doi: http://dx.doi.org/10.1212/WNL.55.5.636. PubMed.
10 Varga Z, Flammer AJ, Steiger P, Haberecker M, Andermatt R, Zinkernagel AS, et al. Endothelial cell infection and endotheliitis in COVID-19. Lancet. 2020;395(10234):1417-8. doi: http://dx.doi.org/ 10.1016/S0140-6736(20)30937-5. PubMed.

11 Watson C, Whittaker S, Smith N, Vora AJ, Dumonde DC, Brown KA. IL-6 acts on endothelial cells to preferentially increase their adherence for lymphocytes. Clin Exp Immunol. 1996;105(1):112-9. doi: http://dx.doi.org/10.1046/j.1365-2249.1996.d01-717.x. PubMed.

12 Magro C, Mulvey JJ, Berlin D, Nuovo G, Salvatore S, Harp J, et al Complement associated microvascular injury and thrombosis in the pathogenesis of severe COVID-19 infection: A report of five cases. Transl Res. 2020;220:1-13. doi: http://dx.doi.org/10.1016/ j.trs1.2020.04.007. PubMed.

13 Menikou S, Langford PR, Levin M. Kawasaki Disease: The Role of Immune Complexes Revisited. Front Immunol. 2019;10:1156. doi: http://dx.doi.org/10.3389/fimmu.2019.01156. PubMed.

14 Licciardi F, Pruccoli G, Denina M, Parodi E, Taglietto M, Rosati S, et al. SARS-CoV-2-Induced Kawasaki-Like Hyperinflammatory Syndrome: A Novel COVID Phenotype in Children. Pediatrics. 2020;146(2):e20201711. doi: http://dx.doi.org/10.1542/peds.2020-1711. PubMed.

15 Craver R, Huber S, Sandomirsky M, McKenna D, Schieffelin J, Finger L. Fatal Eosinophilic Myocarditis in a Healthy 17-Year-Old Male with Severe Acute Respiratory Syndrome Coronavirus 2 (SARS-CoV-2c). Fetal Pediatr Pathol. 2020;39(3):263-8. doi: http://dx.doi.org/10.1080/ 15513815.2020.1761491. PubMed.

16 Yokouchi Y, Oharaseki T, Harada M, Ihara F, Naoe S, Takahashi K. Histopathological study of lymph node lesions in the acute phase of Kawasaki disease. Histopathology. 2013;62(3):387-96. doi: http://dx.doi.org/10.1111/his.12007. PubMed.

17 Luo P, Liu Y, Qiu L, Liu X, Liu D, Li J. Tocilizumab treatment in COVID-19: A single center experience. J Med Virol. 2020;92(7):814-8. doi: http://dx.doi.org/10.1002/jmv.25801. PubMed. 